\title{
Configuration-independent elastic interactions in metal-hydrogen solutions
}

\author{
Ronald Bass $\dagger$, W A Oates $\ddagger$, H R Schober $\S$ and A M Stoneham \\ Theoretical Physics Division, AERE Harwell, Oxon OX11 ORA, UK
}

Received 3 April 1984

\begin{abstract}
We demonstrate quantitative methods for estimating that part of the elastic interaction energy between defects in solids which does not depend on the precise spatial arrangement of the defects. This energy involves both the total volume change per defect in the finite solid and the purely shear part of the volume change. Several different continuum and atomistic modelling methods are used to calculate these volume changes for $\mathrm{H}$ in $\mathrm{Pd}, \mathrm{V}$, $\mathrm{Nb}$ and $\mathrm{Ta}$. An estimate of the configuration-independent elastic interaction energy is made for the $\mathrm{Pd}-\mathrm{H}$ system as a function of the $\mathrm{H}$ concentration, and is given in a form suitable for comparison with statistical models.
\end{abstract}

\section{Introduction}

The early statistical-mechanical calculation on the Pd:H system by Lacher (1937) was based on a lattice-gas model in which pairwise attractive interactions were assumed between nearest-neighbour hydrogens. Subsequently Brodowsky (1966) suggested that a direct elastic interaction was responsible for these empirical attractive interactions. Alefeld (1972) noted, however, that there were also indirect effects in a deformable lattice with a stress-free surface. These indirect attractive terms are configuration independent, i.e., they do not depend on the precise arrangement of the hydrogens relative to one another, and they make no contribution to solute correlations. Nevertheless, these configurationindependent interactions can be a substantial fraction of the total elastic energy, and they should be subtracted from experimental data before comparisons are made with results from model calculations of the configuration-dependent terms. When comparing model calculations with experiment one should not ignore the corresponding purely electronic term which would exist even in a rigid lattice. This comes from the hydrogen-induced shift in the Fermi level which will itself lead to both a volume change and a modification of the defect forces giving the elastic interaction (Stoneham 1983a, b).

The origin of these indirect configuration-independent elastic interaction terms can be seen readily. When a hydrogen enters a lattice with a stress-free surface, it causes a volume change $\Delta V$. This $\Delta V$ is the sum of a contribution $\Delta V_{\mathrm{D}}$ dilating the crystal as a whole and a contribution $\Delta V_{\mathrm{S}}$ from shear alone (Eshelby 1955, 1956). The dilation works against the defect forces of other hydrogens already present, so an indirect interaction energy $E_{\mathrm{I}}$ per

† Permanent address: Department of Physics and Astronomy, University of Kansas, Lawrence, Kansas 66045 , USA.

$\ddagger$ Permanent address: Metallurgy Department. University of Newcastle, Australia.

§ Permanent address: IFF, KFA Jülich. D-5170 Jülich, West Germany. 
pair of hydrogens results. Simple arguments (e.g., that $\Delta V$ is the result of a defect 'pressure' $p_{\mathrm{D}} \equiv-B \Delta V / \Omega_{\mathrm{T}}$, where $B$ is the isothermal bulk modulus and $\Omega_{\mathrm{T}}$ is the crystal volume, so that the work done is $p_{\mathrm{D}} \Delta V_{\mathrm{D}}$ ) show that $E_{\mathrm{I}}=-B \Delta V \Delta V_{\mathrm{D}} / \Omega_{\mathrm{T}}$. The negative sign for $E_{\mathrm{I}}$ implies an attractive interaction between like defects.

Two main descriptions have been used to divide $\Delta V$ into $\Delta V_{\mathrm{S}}$ and $\Delta V_{\mathrm{D}}$. The first is continuum elasticity theory; in this continuum limit $\Delta V_{\mathrm{S}} / \Delta V$ and $\Delta V_{\mathrm{D}} / \Delta V$ are simply functions of the elastic constants. The second approach uses an atomistic description of the host lattice, with pairwise (or possibly more complex) interatomic forces (Horner and Wagner 1974, Wagner 1978, Dietrich and Wagner 1979, Futran et al 1982). In the linear approximation the ratios of the sums over interactions $\left(\Sigma_{a b} W_{a b}^{\infty}\right.$ and $\Sigma_{a b} W_{a b}$ of Horner and Wagner (1974)) define $\Delta V_{\mathrm{S}} / \Delta V$ and $\Delta V_{\mathrm{D}} / \Delta V$. Both approaches have advantages. The continuum method models correctly all the long-range interactions, and (with numerical evaluation of certain integrals) models correctly the changes with temperature or degree of hydrogenation. The lattice methods have the advantage that the configuration-dependent and configuration-independent terms are calculated in a consistent and equivalent way. In the present paper we shall estimate the elastic interactions which depend on the hydrogen concentration but not on its disposition.

\section{Calculations of $\Delta V, \Delta V_{\mathrm{D}}$ and $\Delta V_{\mathrm{S}}$}

In discussing the different volume changes it is convenient (Eshelby 1956) to introduce the coefficients $\gamma$ and $\gamma^{\prime}$ where

$$
\begin{aligned}
& \gamma=\Delta V / \Delta V_{\mathrm{S}} \\
& \gamma^{\prime}=\Delta V_{\mathrm{D}} / \Delta V=1-(1 / \gamma) .
\end{aligned}
$$

Several approaches for calculating the volume change $\Delta V$, the uniform dilatation component $\Delta V_{\mathrm{D}}$ and the pure shear component $\Delta V_{\mathrm{S}}$ are possible. In most of the examples given below we shall refer to $\mathrm{Pd}: \mathrm{H}$, using a nearest-neighbour model potential due to von Heugten (published by Stoneham and Taylor (1981)) which was also adopted by Oates and Stoneham (1983). These provide a convenient reference case, although, for reasons discussed by Oates and Stoneham, one should be cautious of expecting detailed agreement with experiment because of the limitations of the potentials. In other cases we have used the experimental elastic constants of Hsu and Leisure (1979) which are given in table 1. There is clear anisotropy, shown by the deviation from unity of the ratio $2 c_{44} /\left(c_{11}-c_{12}\right)$,

Table 1. Elastic constants and their ratios. Experimental values are from $\mathrm{Hsu}$ and Leisure (1979), 'model' values are from Oates and Stoneham (1983). All elastic constants are in

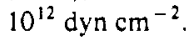

\begin{tabular}{llllll}
\hline & Model & Pd $(0 \mathrm{~K})$ & $\mathrm{Pd}(300 \mathrm{~K})$ & $\mathrm{PdH}_{0.66}(0 \mathrm{~K})$ & $\mathrm{PdH}_{0.66}(300 \mathrm{~K})$ \\
\hline$c_{11}$ & 2.05 & 2.34 & 2.24 & 2.27 & 2.11 \\
$\frac{1}{2}\left(c_{11}-c_{12}\right)$ & 0.31 & 0.29 & 0.25 & 0.33 & 0.27 \\
$c_{44}$ & 0.89 & 0.71 & 0.71 & 0.69 & 0.63 \\
Bulk modulus & 1.64 & 1.95 & 1.90 & 1.83 & 1.75 \\
$\frac{1}{3\left(c_{11}+c_{12}\right)}$ & & & & 2.11 & 2.34 \\
Anisotropy ratio & 2.87 & 2.45 & 2.81 & & \\
$2 c_{44} /\left(c_{11}-c_{12}\right)$ & & & & & \\
\hline
\end{tabular}


and there is a distinct Cauchy violation for $c_{12} \neq c_{44}$. The model potential for Pd gives a similar elastic anisotropy to that observed.

In the presence of significant anisotropy we must go beyond the classic analysis of Eshelby (1956). The critical fact is that if we write the displacements due to a defect in a harmonic crystal of any symmetry in the form

$$
u(r)=u_{\mathrm{D}}(r)+A_{\mathrm{S}} r / r^{3}
$$

where the average over angles of $u_{D}$ only contains forms falling off faster than $r^{-2}$, then the second term leads to a volume change $4 \pi A_{\mathrm{S}}$ but no dilatation. It is the second term, and only this term, which contributes to $\Delta V_{\mathrm{s}}$. This is a mathematical result, unconnected with elasticity theory or any specific assumptions about the elastic constants. Only the term $u_{\mathrm{S}}(\boldsymbol{r})$, proportional to $r / r^{3}$, has the properties of a finite value of $r^{2}(\boldsymbol{r} / r) \cdot \boldsymbol{u}_{\mathrm{S}}(\boldsymbol{r})$ and zero dilatation $\left(u_{x x}+u_{y y}+u_{z 2}\right)$. In practical terms, we should want to estimate $A_{\mathrm{S}}$ at distances not too close to the defect (where parts of $\boldsymbol{u}_{\mathrm{D}}$ may be hard to separate from $\boldsymbol{u}_{\mathrm{S}}$ ) and not too close to any real surface (where the precise topography may matter).

We now consider several different methods for obtaining $\Delta V_{\mathrm{D}}, \Delta V_{\mathrm{S}}, \Delta V$, and their ratios.

\section{1. $\Delta V$ as the pressure derivative of the formation energy}

It is a general result that in a finite crystal the volume of formation is given (Finnis and Sachdev 1976) by

$$
\begin{aligned}
\Delta V & =-\left(\partial U_{\mathrm{F}} / \partial \ln \Omega\right)_{\mathrm{T}} / B \\
& =-\left(\partial U_{\mathrm{F}} / \partial \ln a\right)_{\mathrm{T}} / 3 B
\end{aligned}
$$

where $U_{\mathrm{F}}$ is the formation energy, $B \equiv\left(c_{11}+2 c_{12}\right) / 3$ is the bulk modulus and $a$ is the lattice parameter. $U_{\mathrm{F}}$ is found readily using the Harwell HADES or DEviL codes (see Oates and Stoneham (1983) for details) and the derivative is obtained simply by numerical differentiation. A comment on the potentials is made in the appendix. Values are given in $\S 2.4$.

\section{2. $\Delta V$ from the virial of the defect forces}

Oates and Stoneham (1983) fitted the defect forces $\boldsymbol{F}$ to the experimental result of Peisl (1978), $\Delta V=2.8 \AA^{3}$, using the well known virial form

$$
\begin{aligned}
& \Delta V=\sum_{i, j} \sum_{k} s_{i j k k} G_{i j} \\
& G_{i j}=\sum_{\text {atoms } i} R_{l i} f_{j}\left(R_{l}\right)
\end{aligned}
$$

(Eshelby 1956, Kanzaki 1957, Hardy 1968, Temkin 1970, Stoneham 1975 p 185). One problem results, for equation (5) omits important terms. This has been noted previously for ionic crystals (Flynn 1971, Lidiard 1981, Gillan 1983, Stoneham 1983b) and for metals (Schober 1977, Schober and Ingle 1980, Gillan 1983, Stoneham 1983b). The correction is $\Delta V_{\text {rel }}$ in $\S 2.4$. In the present case $\Delta V_{\text {rel }}$ is only a small correction $\left(0.28 \AA^{3}\right.$ out of $3.05 \AA^{3}$ ). 


\subsection{Values of $\gamma$ and $\gamma^{\prime}$ from elasticity theory}

Isotropic elasticity theory gives directly (see, e.g., Stoneham $1975 \mathrm{p} \mathrm{185)} \mathrm{the} \mathrm{ratios}$

$$
\begin{aligned}
& \gamma=3 c_{11} /\left(c_{11}+2 c_{12}\right) \\
& \gamma^{\prime}=2\left(c_{11}-c_{12}\right) / 3 c_{11}
\end{aligned}
$$

and these expressions lead to the 'isotropic' values of table 2. Liebfried and Breuer (1978) give a list of these for many metals. They also quote expressions appropriate for weak anisotropy. These results have been extended to anisotropic cases by other workers (see, e.g., Dederichs and Pollmann 1972, Liebfried and Breuer 1978). $\Delta V_{\mathrm{S}}$ can be expressed as an integral which is evaluated analytically for small cubic anisotropy $\left(c_{a}=c_{11}-c_{12}-2 c_{44}\right.$ is small). By a variational procedure it has been shown that $\gamma$ can be expressed to first order of the anisotropy factor $c_{a} / c_{44}$ by a formula of the form of equation (7) using the effective isotropic elastic constants (Voigt averages) $\bar{c}_{11}=c_{11}-2 c_{a} / 5, \bar{c}_{12}=c_{12}+c_{a} / 5, \bar{c}_{44}=c_{44}+c_{a} / 5$. For most metals the difference between the exact value of $\gamma$ and the effective isotropic one is less than $5 \%$.

Following Dederichs and Pollmann (1972), the shear component of the volume change of a point defect in a cubic crystal may be calculated from

$$
\begin{aligned}
\Delta V_{\mathrm{S}} & =\int \mathrm{d}^{3} r \operatorname{div} \boldsymbol{u}(\boldsymbol{r}) \\
& =\int_{|\boldsymbol{K}|=1} \frac{\mathrm{d} \Omega}{4 \pi} \boldsymbol{K} \cdot \boldsymbol{u}(\boldsymbol{K}) \\
& =G \int_{|\boldsymbol{K}|=1} \frac{\mathrm{d} \Omega}{4 \pi} \frac{t(\boldsymbol{K})}{1+\left(c_{44}+c_{12}\right) t(\boldsymbol{K})}
\end{aligned}
$$

where $G \delta_{\alpha \beta}$ is the double force tensor and the function $t$ is defined by

$$
t(\boldsymbol{K})=\sum_{\alpha=1}^{3} \frac{K_{\alpha}^{2}}{c_{44}+\left(c_{11}-c_{12}-2 c_{44}\right) K_{\alpha}^{2}} .
$$

Bansil's (1975) 'special directions' method may be used to evaluate this integral, since the integrand has full cubic symmetry. Thirteen directions within the irreducible $\frac{1}{48}$ of the

Table 2. Elasticity theory for $\gamma, \gamma^{\prime}$. Other estimates of $\gamma^{\prime}$ for $\mathrm{Nb}$ are 0.26 (Wagner and Horner 1974) and 0.425 (Zabel and Peisl 1979).

\begin{tabular}{llllll}
\hline FCC & Model & Pd $(0 \mathrm{~K})$ & Pd $(300 \mathrm{~K})$ & $\mathrm{PdH}_{0.66}(0 \mathrm{~K})$ & $\mathrm{PdH}_{0.66}(300 \mathrm{~K})$ \\
\hline$\gamma\left(\Delta V / \Delta V_{\mathrm{S}}\right)$ isotropic & 1.25 & 1.20 & 1.18 & 1.08 & 1.21 \\
Anisotropic & & 1.34 & 1.33 & 1.37 & 1.34 \\
Effective isotropic & 1.52 & 1.43 & 1.43 & 1.47 & 1.43 \\
$\gamma^{\prime}\left(\Delta V_{\mathrm{D}} / \Delta V\right)$ isotropic & 0.20 & 0.17 & 0.15 & 0.07 & 0.17 \\
Anisotropic & & 0.25 & 0.25 & 0.27 & 0.25 \\
Effective isotropic & 0.34 & 0.30 & 0.30 & 0.32 & 0.30 \\
\hline
\end{tabular}

\begin{tabular}{llll}
\hline BCC & $\mathrm{V}$ & $\mathrm{Nb}$ & $\mathrm{Ta}$ \\
\hline$\gamma$ & 1.403 & 1.290 & 1.468 \\
$\gamma^{\prime}$ & 0.287 & 0.225 & 0.319 \\
\hline
\end{tabular}




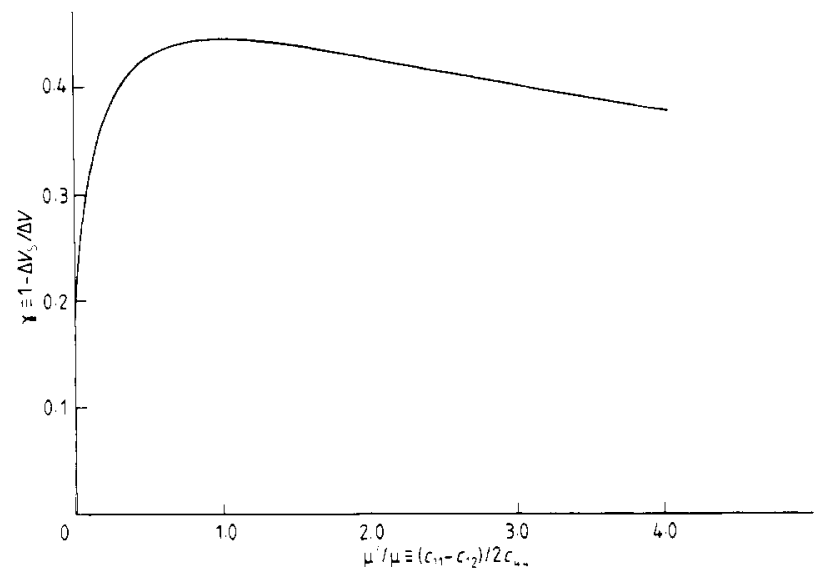

Figure 1. Eshelby's (1956) coefficient $\gamma$ for a cubic crystal obeying the Cauchy relation $c_{12}=c_{44}$. The value of $\gamma$ is given as a function of the ratio $\mu^{\prime} / \mu$ of the two shear moduli $\mu^{\prime} \equiv \frac{1}{2}\left(c_{11}-c_{12}\right)$ and $\mu \equiv c_{44}$.

Brillouin zone were used. Figure 1 shows the results for a Cauchy solid $\left(c_{12}=c_{44}\right)$ for various ratios of the two shear constants $\left(\mu^{\prime} \equiv \frac{1}{2}\left(c_{11}-c_{12}\right)\right) /\left(\mu \equiv c_{44}\right)$. Values for the BCC metals $\mathrm{V}, \mathrm{Nb}, \mathrm{Ta}$ and for $\mathrm{Pd}$ are given in table 2 , together with the effective isotropic values.

\subsection{Calculations of $\Delta V$ by computer simulation}

The double force tensor $G_{i j}$ (equation (6)) can be calculated easily by computer simulation techniques. Two possible techniques have been discussed by Schober and Ingle (1980) and a third method will be discussed later in this section. In the first method $G_{i j}$ is calculated as the moment of the forces exerted by the movable atoms in region $I$, because of their relaxation, on the fixed atoms in the surrounding region II. For pair forces this gives

$$
G_{i j}=\sum_{\substack{m \in \mathbb{I I} \\ n \in \mathrm{I}}} R_{i}^{0 m} F_{j}\left(R^{m}-R^{n}\right)
$$

where $R^{0 n}$ and $R^{n}$ denote the ideal and displaced positions, respectively, of atom $n$. The second method uses the displacements calculated in region I to define generalised Kanzaki forces $K$ which contain the contributions of the host lattice anharmonicity:

$$
K_{i}^{m}=\sum_{n i} \varphi_{i j}^{m n}\left(R_{j}^{n}-R_{j}^{0 n}\right)
$$

where $\varphi$ is the ideal lattice harmonic-coupling matrix and

$$
G_{i j}=\sum_{m \in \mathrm{I}} R_{i}^{0 m} K_{j}^{m}
$$

Both methods converge rapidly with increasing number of atoms $N_{\mathrm{I}}$ in region I but require a higher-accuracy energy minimisation than is needed if one is interested in the energies only, in common with all methods for calculating $G$ or $\Delta V$. For the model of $\mathrm{H}$ in $\mathrm{Pd}$ considered here we have calculated a value of $G_{i j}=2.85 \delta_{i j} \mathrm{eV}$, and hence $\Delta V=2.77 \AA^{3}$. Using the zeroth-order formula (11) we would have obtained $G_{i j}^{0}=3.13 \delta_{i j} \mathrm{eV}$ and 
$\Delta V^{0}=3.05 \AA^{3}$ instead. The relaxation of the Pd lattice therefore causes an anharmonicity correction $\Delta V_{\text {rel }}=-0.28 \AA^{3}$. With the 'effective' isotropic value of $\gamma$ we obtain $\Delta V_{\mathrm{S}}=1.82 \AA^{3}$.

We can also exploit the explicit volume dependence of the model to calculate $\gamma$ by computer simulation with elastic boundary conditions. The energy of the crystal is given by

$$
E=\frac{1}{2} \sum_{R R^{\prime}} \varphi\left(R-R^{\prime}\right)+p_{\mathrm{c}} V
$$

where $p_{\mathrm{c}}=\frac{1}{2}\left(c_{12}-c_{44}\right)=0.178 \mathrm{eV} \AA^{-3}$ is the Cauchy pressure. We now impose continuum theoretical displacements on the atoms in region II. The energy change in region I can be expanded in a power series in $\operatorname{Tr} G$ (note $G$ is isotropic in our case) as

$$
\Delta E=\varepsilon_{1}(\operatorname{Tr} G)+\varepsilon_{2}(\operatorname{Tr} G)^{2}+\ldots
$$

The equilibrium condition of the ideal lattice gives

$$
\varepsilon_{1}(\operatorname{Tr} G)=-p_{\mathrm{c}} \Delta V_{\mathrm{S}}
$$

and hence $\gamma$ and $\varepsilon_{1}$ are related by the expression

$$
\gamma=-\frac{3 B}{p_{\mathrm{c}}} \varepsilon_{1} .
$$

We have evaluated this formula for a number of crystal sizes and geometries (see table 3 ). The anisotropic elastic continuum model displacements of atoms in region II were calculated by a one-dimensional numerical integration routine (Deutz and Schober 1983). Region I was taken either as cubic with the dilatation centre at $(0.5 a, 0,0)$ or as spherical with the dilatation centre at the origin. To estimate the lattice surface effects, $\Delta E$ included either the full I-II bonds $\left(\Delta E_{1}\right)$ or half the bonds $\left(\Delta E_{2}\right)$. The resulting values of $\gamma$ are compiled in table 2. The difference in the $\gamma$ values obtained from $\Delta E_{1}$ and $\Delta E_{2}$ shows the importance of I-II cross bonding. There is also a pronounced dependence on the shape of region I and a less pronounced dependence on its size. This has to be expected since, due to lattice anharmonicity, a point-dipole force is smeared out over a large area (Schober 1977). The difference between the values of $\gamma$ including a single $\mathrm{H}$ defect and those excluding it is less than 0.01 , showing that $\gamma$ is indeed a host lattice property as predicted by theory.

Another method for calculating $\Delta V_{S}$ has been used by Seeger and Mann (1960) and Johnson (1964). They make an effective isotropic ansatz for the displacement field in

\begin{tabular}{|c|c|c|c|}
\hline Model & $N_{\mathrm{I}}$ & From $\Delta E_{\mathfrak{l}}$ & From $\Delta E_{2}$ \\
\hline Effective isotropic value & & 1.52 & 1.52 \\
\hline \multirow[t]{2}{*}{ Cubic inner region } & 655 & 1,46 & 1.65 \\
\hline & 1687 & 1.41 & 1.56 \\
\hline \multirow[t]{2}{*}{ Spherical inner region } & 466 & 1.31 & 1.53 \\
\hline & 682 & 1.30 & 1.50 \\
\hline
\end{tabular}
region II,

$$
u(r)=\left(\Delta V_{\mathrm{S}} / 4 \pi\right)\left(r / r^{3}\right)
$$

Table 3. Values of $\gamma$ deduced from equation (17). 
Table 4. Summary of $\Delta V$ estimates for $\mathrm{H}$ at octahedral sites of $\mathrm{Pd}$. The forces were fitted by Oates and Stoneham (1983) using $B=18.05 \times 10^{10} \mathrm{~Pa}$, whereas the virial estimate is obtained using $B=16.40 \times 10^{10} \mathrm{~Pa}$ consistent with the potential.

\begin{tabular}{ll}
\hline & $\Delta V\left(\AA^{3}\right)$ \\
\hline Experiment & 2.9 \\
Virial $\Delta V$ & 3.05 \\
$-\left(\partial U_{\mathrm{F}} / \partial \ln \Omega\right) / B$ & 2.77 \\
$\Delta V_{\mathrm{S}}$ & 1.82 \\
$\Delta V_{\text {Rel }}$ & -0.28 \\
\hline
\end{tabular}

and $\Delta V_{\mathrm{S}}$ is calculated as a variational parameter in addition to the displacements of the ions in region $I$. The total energy for this ansatz is given by

$$
\Delta E=\frac{1}{2} \sum_{m \in \mathrm{I}} \sum_{n \in \mathrm{I}, \mathrm{II}} \varphi\left(R^{m}-R^{n}\right)+b\left(\Delta V_{\mathrm{S}}\right)^{2}
$$

where the last term is the elastic energy stored in the infinite crystal outside region I. For a spherical region I (of volume $V_{\mathrm{I}}$ ) the constant $b$ can be calculated easily (Johnson 1964) as

$$
b=\frac{2}{3}\left[c_{44}+\frac{1}{3}\left(c_{11}-c_{12}\right)\right] / V_{1} .
$$

It should be noted that $V_{1}$, the volume of the inner region, is not a well defined quantity and the inner region is never completely spherical for a finite $N_{\mathrm{I}}$. Nevertheless Johnson's results minimising equation (18) agree quite well with the results given by Dederichs et al (1978), who calculated the dipole force tensor by the surface force and extended Kanzaki force (1957) models. In the present case the situation is much worse since $\Delta V_{\mathrm{S}}$ is much smaller. This results in the linear term in equation (18) being three orders of magnitude larger than the quadratic dependence on $\Delta V_{\mathrm{S}}$ in the first and third terms. The linear second term should cancel exactly with the linear $\Delta V_{\mathrm{S}}$ dependence of the first term. This cancellation can be made simply for energy calculations (Schober 1983) by comparison with ideal crystal calculations with the same boundary conditions. For calculations of $\Delta V_{\mathrm{S}}$ this is also possible, but very tedious. By very careful minimisation of equation (18) we gained a value of $\gamma=1.57$. The good agreement with the values in table 4 is perhaps fortuitous since a change in energy of $10^{-5} \mathrm{eV}$ causes a change of $15 \%$ in $\Delta V_{\mathrm{s}}$ and hence in $\gamma$. If we count only half the I-II bonds towards the first term we find no minimum at all with respect to $\Delta V_{\mathrm{s}}$. We may conclude that the determination of $\Delta V_{\mathrm{S}}$ by direct energy minimisation is highly unreliable, especially for systems with small $\Delta V$ and large $p_{\mathrm{c}}$. Whilst the other methods, based on surface forces or generalised Kanzaki forces, can become inaccurate, they cannot fail catastrophically like the direct energy minimisation.

\subsection{Other methods}

The most simple atomistic method, though regrettably unreliable, is to look at values of $4 \pi r(\boldsymbol{r} \cdot \boldsymbol{u})$ from a typical HADES runt. Here $\boldsymbol{u}$ is the outward normal displacement of atoms at distance $r$ from the interstitial. The problems are clear: at small $r$ various small shortrange effects confuse the results and at large $r$ the displacements are constrained to fall to zero at a chosen distance. We plot some results in figure 2 for illustrative purposes. Clearly this approach to $\Delta V_{\mathrm{s}}$ is not too useful. 

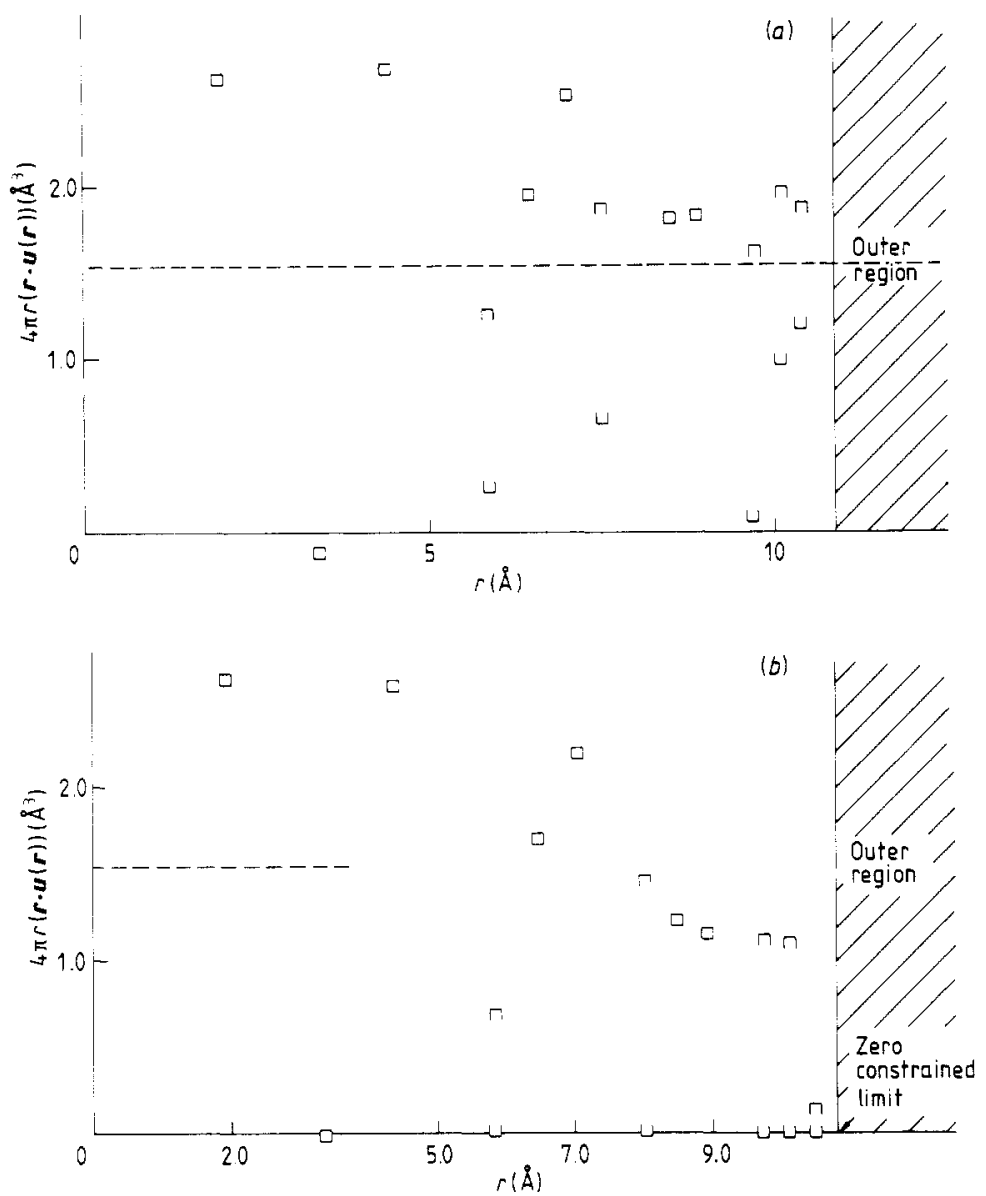

Figure 2. Values of $\Delta V_{\mathrm{S}}$ as deduced from the displacements of shells of atoms. The values plotted are $4 \pi r(\boldsymbol{r} \cdot \boldsymbol{u})$ where $\boldsymbol{u}$ is the outward normal displacement of the shell initially at $r$. Note that for some values of $r$ there are two inequivalent shells of atoms, and these are both indicated. (a) The displacements in the outer region have the form $A_{\mathrm{s}} r / r^{3}$ with chosen $A_{\mathrm{S}}$; the value as constrained in the outer region is given $(---)$. (b) $A_{\mathbf{S}}$ is zero; the value for the relaxed outer region is shown (- - ). The fall-off at large $r$ results from the constraint $u=0$ outside the 267 atoms explicitly relaxed.

\section{The configuration-independent interaction energy}

In terms of the Eshelby coefficient $\gamma^{\prime}$ (equation (2)) the non-configurational interaction energy per pair, $E_{1}$, may be written

$$
E_{\mathrm{I}}=-\gamma^{\prime} B(\Delta V)^{2} / \Omega
$$

Suppose there are $N$ defects present and that the non-configurational interaction energy is $E_{\mathrm{I}}$ per pair. If $E_{\mathrm{I}}$ is independent of $N$ then the total energy from these interaction is $E_{\mathrm{NC}}(N)$ where

$$
E_{\mathrm{NC}}(N)=\frac{1}{2} N(N-1) E_{\mathrm{I}} \simeq \frac{1}{2} N^{2} E_{\mathrm{I}} .
$$


We can also define an equivalent chemical potential $\mu_{\mathrm{NC}}(N)$ as

$$
\begin{aligned}
\mu_{\mathrm{NC}}(N) & =\frac{\partial}{\partial N} E_{\mathrm{NC}}(N) \simeq N E_{\mathrm{I}} \\
& =-\gamma^{\prime} B(\Delta V)^{2}\left(\theta / V_{\mathrm{S}}\right) \\
& =-\gamma^{\prime} B(\Delta V)^{2}(x / \Omega) .
\end{aligned}
$$

Here $V_{\mathrm{S}}$ is the volume per hydrogen site, $\Omega$ is the atomic volume of the host and, as measures of concentration, $x \equiv$ hydrogen/metal ratio and $\theta \equiv$ fractional occupancy of the interstitial sites. $\Delta V$ is, of course, the partial molar volume of hydrogen.

If we assume that $E_{\mathrm{I}}$ varies slowly with $N$, i.e.

$$
E_{\mathrm{l}}(N)=E_{\mathrm{I}}(0)+\left(\mathrm{d} E_{\mathrm{l}} / \mathrm{d} N\right) N,
$$

then we find that

$$
\begin{aligned}
E_{\mathrm{NC}}(N) & \sim \frac{1}{2} N^{2} E_{\mathrm{I}}(0)+\frac{1}{3} N^{3}\left(\mathrm{~d} E_{\mathrm{I}} / \mathrm{d} N\right) \\
& \sim \frac{1}{2} N^{2} \bar{E}
\end{aligned}
$$

where we introduce $\bar{E}=\frac{1}{3}\left(E_{\mathrm{I}}(0)+2 E_{\mathrm{I}}(N)\right)$ as the appropriate average energy. For the chemical potential, if we ignore terms in $\mathrm{d}^{2} E_{\mathrm{I}} / \mathrm{d} N^{2}$, we have

$$
\begin{aligned}
\mu_{\mathrm{NC}}(N) & \sim N E_{\mathrm{l}}(0)+N^{2}\left(\mathrm{~d} E_{\mathrm{l}} / \mathrm{d} N\right) \\
& \sim N E_{\mathrm{l}}(N) .
\end{aligned}
$$

In principle, this equation could be used in conjunction with equation (20), but there remains the problem of knowing $\gamma^{\prime}$ as a function of $\mathrm{H}$ concentration. Of the methods outlined in $\S 2$, only the isotropic continuum calculations can be used (see table 2 for $\left.\mathrm{PdH}_{0.66}\right)$.

An alternative approach to obtaining the non-configurational contribution at high $\mathrm{H}$ concentration is to consider the difference between the total and configuration-dependent interaction energies.

The total elastic interaction energy, $E_{\mathrm{T}}$, is known exactly for an arbitrary lattice of any shape (see Wagner and Horner 1974, equation 4.44):

$$
E_{\mathrm{T}}=-\frac{1}{2} B(\Delta V)^{2} \theta^{2} / V_{\mathrm{S}}
$$

Oates and Stoneham (1983) have demonstrated that the configurational-dependent interaction energy, $E_{\mathrm{C}}$, depends on both the arrangement and number of $\mathrm{H}$ atoms but, to first order, we might expect a simple analytical form for the composition dependence if the $\mathrm{H}$-atom distribution is random. In the case of random substitutional solid solutions, the local relaxation energy varies roughly as $\theta(1-\theta)$ (Flynn 1972, Froyen and Herring 1981). Since the relaxation energy must also be zero at $\theta=0$ and $\theta=1$ for interstitial solid solutions, we may write

$$
E_{\mathrm{C}}=-\tilde{A} \theta(1-\theta)
$$

where $\tilde{A}$ is a parameter to be determined.

From the difference $E_{\mathrm{T}}-E_{\mathrm{C}}$ we obtain the non-configurational energy and, on comparing this result with that obtained from a combination of equations (20) and (21) for 
the dilute solution, the value of $\tilde{A}$ is obtained as

$$
\tilde{A}=\frac{1}{2} B \Delta V_{2} \theta\left(1-\gamma_{0}^{\prime}\right) / V_{\mathrm{S}}
$$

where $\gamma_{0}^{\prime}$ is the value of $\gamma^{\prime}$ in the very dilute solution. Hence for this special case of a random distribution of $\mathrm{H}$ atoms

$$
\begin{aligned}
& E_{\mathrm{NC}}=\left[\gamma_{0}^{\prime}+\left(1-\gamma_{0}^{\prime}\right) \theta\right] E_{\mathrm{T}} \\
& E_{\mathrm{C}}=\left(1-\gamma_{0}^{\prime}\right)(1-\theta) E_{\mathrm{T}}
\end{aligned}
$$

so that $\gamma^{\prime}=\left[\gamma_{0}^{\prime}+\left(1-\gamma_{0}^{\prime}\right) \theta\right]$ becomes unity when all the sites are occupied, i.e., all the elastic energy is non-configurational.

Taking $V_{\mathrm{S}}=8.87 \mathrm{~cm}^{3} \mathrm{~mol}^{-1} \quad$ (Pearson 1958), $\Delta V / \Omega=0.19$ (Peisl 1978), $B=1.90 \times 10^{11} \mathrm{~Pa}, \mathrm{~d} B / \mathrm{d} \theta=-0.232 \times 10^{11} \mathrm{~Pa}$ (Hsu and Leisure 1979) and $\gamma_{0}^{\prime}=0.25$ for $\mathrm{Pd}-\mathrm{H}$ alloys at $300 \mathrm{~K}$, equations (27)-(31) may be differentiated, as in (22), to give the total $\left(\mu_{\mathrm{T}}\right)$, configurational $\left(\mu_{\mathrm{C}}\right)$, and non-configurational $\left(\mu_{\mathrm{NC}}\right)$ elastic energy contributions

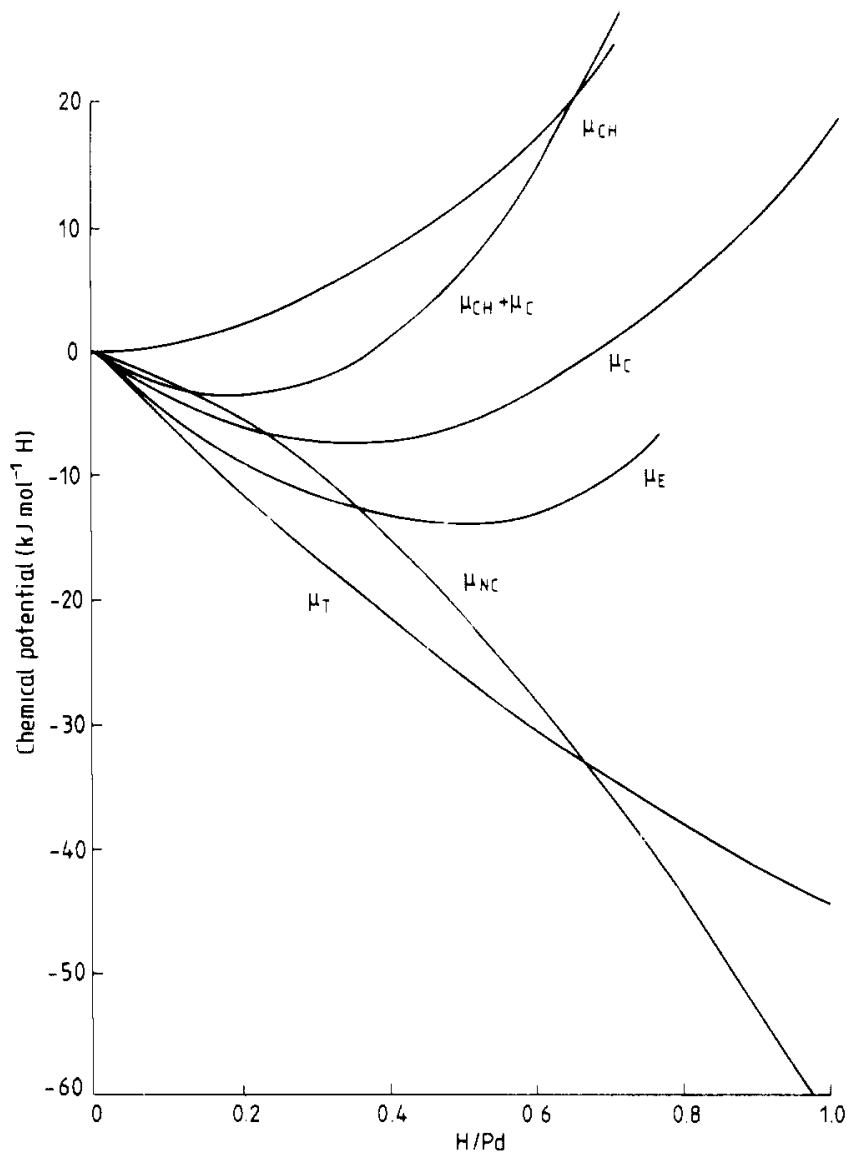

Figure 3. Contributions to the chemical potential for hydrogen in $\mathrm{Pd}$ over a range of hydrogen concentrations. The total chemical potential in elasticity theory is $\mu_{\mathrm{T}}$ with configurational part $\mu_{\mathrm{C}}$ (equations (27) and (31)) and non-configurational part $\mu_{\mathrm{NC}}$ (equations (27) and (30)). The experimental excess chemical potential, $\mu_{E}$, is the sum of the total elastic part, $\mu_{\mathrm{T}}$, and a chemical part, $\mu_{\mathrm{CH}}$. The combination of terms appropriate for statistical calculations is $\mu_{\mathrm{CH}}+\mu_{\mathrm{C}}$ which is also shown. 
to the chemical potential. The difference $\mu_{\mathrm{E}}-\mu_{\mathrm{T}}$, where $\mu_{\mathrm{E}}$ is the experimental excess chemical potential (Kuji et al 1983), is the chemical contribution $\mu_{\mathrm{CH}}$ to the chemical potential. All these quantities are shown in figure 3 for the $\mathrm{Pd}-\mathrm{H}$ system at $300 \mathrm{~K}$. It is of interest to note that the effect of $\mathrm{H}$ concentration on $\mu_{\mathrm{CH}}$ is quite different from that expected from simple arguments based on $\mathrm{d}$ - and s-band filling. Rather, a strong repulsive interaction between $\mathrm{H}$ atoms is indicated. Statistical models should be concerned with the sum $\mu_{\mathrm{CH}}+\mu_{\mathrm{C}}$ of the contributions resulting from the direct interactions which is also shown in figure 3. It has been noted previously (Oates and Stoneham 1983) that for $\mu_{\mathrm{C}}$, at least, a pairwise interpretation is inadequate.

\section{Appendix. Interatomic potentials}

The Pol potential we use is almost exactly that of Van Heughten (listed as his potential I by Stoneham and Taylor (1981)). The small changes we make ensure a smooth cut-off at $r_{\mathrm{c}}=3.305 \AA$. The coefficient $A_{1}$ in $V(R)=\Sigma_{n=1}^{6} A_{n} R^{n}$ changes from -396.6696 to -396.6861 , with a similar small change in $A_{0}$ to give $V\left(r_{c}\right)=0$. As can be seen from table 5 , there is no significant change in the elastic constants, and we believe the situation will be essentially the same for all but special quantities. Our experience has emphasised how sensitive one must be in making approximations to these potentials, so the agreement indicated is important.

The total energy of the crystal can be written as

$$
E=\frac{1}{2} \sum_{R, R^{\prime}} V\left(R-R^{\prime}\right)+p_{\mathrm{c}} V
$$

where $V$ is the crystal volume and $p_{c}$ is the Cauchy pressure

$$
p_{\mathrm{c}}=\frac{1}{2}\left(c_{12}-c_{44}\right)=0.178 \mathrm{eV} \AA^{-3}=10.33 \mathrm{eV} \mathrm{a}^{-3}
$$

where the lattice constant $a=3.872 \AA$.

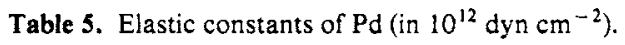

\begin{tabular}{llll}
\hline & Original $A_{0}, A_{1}$ & Modified $A_{0}, A_{1}$ & Experiment \\
\hline$c_{11}$ & 2.05 & 2.04 & 2.26 \\
$c_{12}$ & 1.43 & 1.45 & 1.76 \\
$c_{44}$ & 0.89 & 0.88 & 0.72 \\
$\frac{1}{2}\left(c_{11}-c_{12}\right)$ & 0.31 & 0.30 & 0.25 \\
\hline
\end{tabular}

\section{References}

Alefeld G 1972 Ber. Bunsenges Phys. Chem. 76355

Bansil A 1975 Solid State Commun. $16885-9$

Brodowsky H 1966 Z. Phys. Chem., NF 44129

Dederichs P H, Lehmann C, Schober H R, Scholz A and Zeller R 1978 J. Nucl. Mater. 69-70 176

Dederichs P H and Pollmann J 1972 Z. Phys. 255315

Deutz J W and Schober H R 1983 Comput. Phys. Commun. 3087

Dietrich S and Wagner H 1979 Z. Phys. B 36121

Eshelby J 1955 Acta Metall. 3487

1956 Solid State Phys. 3487 (New York: Academic) 
Finnis M W and Sachdev M 1976 J. Phys. F: Met. Phys. 6965

Flynn C P 1971 Z. Naturf. a 2599

- 1972 Point Defects and Diffusion (Oxford: University Press)

Froyen J S and Herring C 1981 J. Appl. Phys. 527165

Futran M, Oats S G, Hall C K and Welch D O 1982 J. Chem. Phys. 776223

Gillan M J 1983 Phil. Mag. A 48903

Hardy J R 1968 J. Phys. Chem. Solids 292009

Horner H and Wagner H 1974 J. Phys. F: Met. Phys. 73305

Hsu D K and Leisure R G 1979 Phys. Rev. B 201342

Johnson R A 1964 Phys. Rev. 134 A 1329

Kanzaki H 1957 J. Phys. Chem. Solids 227

Kuji T, Oates W A, Bowerman B S and Flanagan T B 1983 J. Phys. F: Met. Phys. 131785

Lacher J R 1937 Proc. R. Soc. A 161525

Liebfried G and Brever N 1978 Point Defects in Metals I, Springer Tracts in Modern Physics 81 (Berlin: Springer)

Lidiard A B 1981 Phil. Mag. 43291

Norgett M J 1974 AERE Report $R-7650$

Oates W A and Stoneham A M 1983 J. Phys. F: Met. Phys. 132427

Pearson W B 1958 Handbook of Lattice Spacings (New York: Pergamon)

Peisl H 1978 Hydrogen in Metals, Springer Topics in Applied Physics vol. 28 ed. G Alefeld and J Völkl (Berlin: Springer) p 70

Schober H R 1977 J. Phys. F: Met. Phys. 4351

- 1983 Radiat. Eff. Lett. 7619

Schober H R and Ingle K W 1980 J. Phys. F: Met. Phys. 10575

Seeger A and Mann E 1960 J. Phys. Chem. Solids 12326

Stoneham A M 1975 Theory of Defects in Solids (Oxford: University Press)

1983a Electronic Structure and Properties of Hydrogen in Metals ed. P Jena and C Satterthwaite (New York: Plenum) p 665

1983b J. Phys. C: Solid State Phys. 16 L925

Stoneham A M and Taylor R J 1981 Handbook of Interatomic Potentials II, AERE Report R-10205

Temkin D E 1970 Sov. Phys.-Solid State 111614

Wagner H 1978 in Hydrogen in Metals, Springer Topics in Applied Physics vol. 28 ed. G Alefeld and J Völk! (Berlin: Springer) p 5

Wagner $\mathrm{H}$ and Horner $\mathrm{H} 1974$ Adv. Phys. 23588

Zabel H and Peisl H 1979 J. Phys. F: Met. Phys. 91461 